framum

Sociológico

\section{Forum Sociológico}

Série II

17 | 2007

Envelhecimento activo. Um novo paradigma

\title{
La importancia del capital social y la participación ciudadana en los planes de rehabilitación : los casos de Ferrol Vello y la Magdalena
}

\section{Romina Prado-Fernández}

\section{(2) OpenEdition}

Edición electrónica

URL: https://journals.openedition.org/sociologico/1706

DOI: 10.4000/sociologico.1706

ISSN: 2182-7427

Editor

CICS.NOVA - Centro Interdisciplinar de Ciências Sociais da Universidade Nova de Lisboa

Edición impresa

Fecha de publicación: 1 enero 2007

Paginación: 103-115

ISSN: 0872-8380

\section{REFERENCIA ELECTRÓNICA}

Romina Prado-Fernández, «La importancia del capital social y la participación ciudadana en los planes de rehabilitación : los casos de Ferrol Vello y la Magdalena», Forum Sociológico [En línea], 17 | 2007, Puesto en línea el 01 enero 2007, consultado el 30 marzo 2022. URL: http://journals.openedition.org/sociologico/1706 ; DOI: https://doi.org/10.4000/sociologico.1706 


\title{
LA IMPORTANCIA DEL CAPITAL SOCIAL Y LA PARTICIPACIÓN CIUDADANA EN LOS PLANES DE REHABILITACIÓN: LOS CASOS DE FERROL VELLO Y LA MAGDALENA*
}

\author{
Romina Prado Fernández \\ Universidad de A Coruña (UDC); (roprafer@yahoo.es)
}

\begin{abstract}
Resumen
A lo largo de los últimos veinte años han sido desarrollados varios proyectos de rehabilitación urbana en España, con el fin de proteger el patrimonio arquitectónico de nuestras ciudades. En este artículo presentaremos algunos datos preliminares que han sido obtenidos en el contexto de un proyecto de investigación colectivo sobre políticas urbanas de rehabilitación en los barrios históricos de Ferrol Vello y la Magdalena.

Nuestra hipótesis es que este plan de rehabilitación está alcanzando notables éxitos en la persecución de sus objetivos fundamentales, debido en gran medida a la existencia de un importante capital social, como resultado y precondición de la participación ciudadana. La adopción de una metodología basada en la combinación de técnicas cualitativas y cuantitativas -intentando ir más allá de la presentación de meros datos, aportando una mayor profundidad en las interpretacionesnos permite argumentar a favor del relativo éxito del mencionado plan de rehabilitación.
\end{abstract}

Palabras clave: Vivienda, capital social, políticas sociales, rehabilitación

\section{Abstract}

For the last twenty years, several urban rehabilitation projects have been developed in Spain, aiming at protecting our cities' architecture heritage. In this paper we present some preliminary data obtained from a collective research project on the urban rehabilitation policies in the ancient quarters of Ferrol Vello ("Old Ferrol") and La Magdalena.

My hypothesis is that this rehabilitation program has been highly effective achieving its main goals, due to a great extent to the existence of an important social capital, as a result and a precondition of citizen participation. The choice for a combined methodology based on qualitative and quantitative techniques - as we try to go beyond the presentation of raw data, providing deeper interpretations - allows us as to argue positively in favour of the aforementioned rehabilitation program.

Keywords: Housing, social capital, social policies, urban rehabilitation

\section{Resumo}

Durante os últimos 20 anos foram desenvolvidos numerosos projectos de reabilitação urbana em Espanha, com a finalidade de proteger o património arquitectónico das nossas cidades. Neste artigo, apresentaremos alguns dados preliminares, obtidos no contexto de um projecto de investigação colectivo sobre políticas urbanas de reabilitação nos bairros históricos de Ferrol Vello e la Magdalena.

A nossa hipótese é que este plano de reabilitação está a alcançar um êxito notável na realização dos seus objectivos fundamentais, devido, sobretudo, à existência de um importante capital social, como resultado e pré-requisito da participação cidadã. A adopção de uma metodologia baseada na combinação de técnicas quantitativas e qualitativas - tentado ir para além da mera apresentação de dados, e trazendo uma maior profundidade às interpretações - permite-nos argumentar em favor do êxito relativo do referido plano de reabilitação.

Palavras-chave: Habitação, capital social, políticas sociais, reabilitação

\footnotetext{
* El presente artículo supone una revisión del póster presentado en el IX Congreso Español de Sociología (Barcelona, 13-15 de septiembre de 2007): "La rehabilitación en Ferrol: la importancia del capital social y la participación ciudadana en la rehabilitación de Ferrol Vello y la Magdalena". Trabajo insertado en el proyecto de investigación << Patrimonio de futuro: Investigación Acción Participativa en la Ciudad de Ferrol> >, que ha recibido financiación, código PGIDIT04CS0102011PR, de la Secretaría de Investigación y Desarrollo de la Xunta de Galicia durante los años 2004-2006
} 


\section{Introducción}

Abordar el análisis de una política social y los elementos que pueden llegar a interactuar en el éxito o fracaso de la misma, como es el caso de la política de rehabilitación de los barrios históricos de Ferrol Vello y la Magdalena, nos ha llevado a plantearnos muy diversas cuestiones en relación al entorno más amplio en que se inserta la ciudad objeto de análisis. Así, nos planteamos cuestiones relativas a la evolución de estas políticas en nuestra historia, las agencias/agentes que intervienen de forma directa 0 indirecta en su marcha, etc., dando lugar a multitud de preguntas difíciles de responder para las cuales, dado su enraizamiento en las más diversas esferas de la sociedad estudiada, se ha hecho necesaria la adopción de una metodología basada tanto en técnicas cuantitativas como cualitativas.

En el planteamiento inicial nos centramos principalmente en dos hipótesis íntimamente relacionadas entre sí, a saber, que el Plan de Rehabilitación estaba consiguiendo notables éxitos en la persecución de sus objetivos fundamentales y que, en gran medida, ese éxito sería debido a la existencia de un fuerte capital social que fomentaría, y sería fomentado, por la participación ciudadana. Más concretamente, argumentamos que es la existencia de una fuerte tradición de capital social en el contexto social estudiado un elem ento que adquiere notable importancia en la implicación de la población en la marcha del plan, llegando a producirse situaciones en las cuales se generan líderes que hacen resaltar aún más dicha participación.

Ilustrativo resulta, en este sentido, la cercanía temporal entre las primeras movilizaciones contra la destrucción de lugares históricamente emblemáticos de las ciudades (como serían los casos del Covent Garden en Londres o La Corrala en Madrid, entre otros) en los años setenta y la aparición del tema de la participación ciudadana en la legislación internacional relativa a la conservación y protección del patrimonio histórico en general, y los centros históricos en particular.

A fin de establecer unas primeras coordenadas para entender el desarrollo de las políticas de rehabilitación dentro de la evolución de las ciudades en nuestra historia más reciente, dedicaremos una primera parte al análisis de las políticas de rehabilitación en los dos ámbitos de mayor envergadura en que se ubica la ciudad de Ferrol, la comunidad autónoma gallega y el estado español. Presentando a continuación las características básicas del plan estudiado, con lo que nos podremos adentrar en el tratamiento del tema del capital social como elemento favorecedor en la consecución de los objetivos marcados que está teniendo el plan de rehabilitación.
Rehabilitación y modelo de ciudad: la intervención en los cascos históricos

La situación de oposición entre renovación y rehabilitación se puso ya de relieve en las primeras manifestaciones de los movimientos sociales en el contexto europeo a partir de los años setenta, aunque podemos mencionar como una posible precursora de este tipo de políticas el desarrollo de una política de rehabilitación (y protección) del patrimonio histórico edificado materializada en la promulgación en Francia de la denominada Ley Malraux (1962), que dio lugar a la creación de la categoría de los Sectores Salvaguardados y Zonas de Protección del Patrimonio Arquitectónico, Urbano y Paisajístico (ZPPAUP), quizá influyendo en cierto modo en la legislación internacional europea y supranacional posterior. La oposición a la renovación urbana tuvo como justificación las desfavorables consecuencias sociales introducidas por el desarrollo de este tipo de intervenciones para los habitantes de estas zonas. Mas la intervención a través de la rehabilitación no estaría tampoco exenta de consecuencias negativas para la población residente en estas zonas, al entrar en juego el riesgo de que, con el cambio de estatus de estas zonas en la configuración de la ciudad, se dé un proceso de "gentrification", esto es, una expulsión de las capas más desfavorecidas y el aburguesamiento de la zona por el efecto de la especulación.

En España, la intervención de los entes públicos en el desarrollo de los planes de rehabilitación deriva de reales decretos que acompañan a cada uno de los Planes de vivienda del Estado, los cuales versan principalmente sobre las medidas de financiación, estableciendo los requisitos para poder acceder a las ayudas concedidas para dicho fin, como serían los préstamos cualificados y las ayudas directas: subsidiación de préstamos cualificados, subvenciones y otras ayudas directas. En este sentido, podemos situar el nacimiento de las políticas de rehabilitación en la promulgación del Decreto-Ley 12/1980, que contempla la rehabilitación de viviendas como materia de protección oficial por parte del Estado, al cual seguirían tres decretos posteriores (375/1982, $2555 / 1982$ y $2329 / 1983$ ) que introdujeron mayores desarrollos de la concesión de ayudas para este fin, presentando mejoras en el establecimiento de las normas que habrían de regir esta concesión de ayudas.

Aparece así la figura de las Áreas de Rehabilitación Integral (ARI), las cuales son zonas o barrios que se encuentran en un estado de degradación tal que, tras la realización de los estudios pertinentes, son declaradas como tales a fin de ser objeto de actuaciones de rehabilitación. Sin embargo, el reconocimiento de las Áreas de Rehabilitación Integral en la legislación, y en las políticas sociales 
desarrolladas por el Instituto Galego de Vivenda e Solo (en adelante IGVS) ${ }^{1}$, tardaría bastantes años en llegar, al centrarse la mayoría de los desarrollos legislativos gallegos sobre rehabilitación en actuaciones en el ámbito rural, al no contemplarse el caso de los conjuntos de interés arquitectónico o patrimonial hasta una orden de 1989 (Diario Oficial de Galicia del 4 de abril de 1989).

A partir de esta legislación estatal, las comunidades autónomas desarrollan también sus propios planes de vivienda y firman convenios con el Ministerio de Vivienda, los cuales posibilitarían, previo acuerdo con el Ayuntamiento respectivo, la puesta en marcha de planes como el de Ferrol Vello y la Magdalena, esto es, la intervención sobre áreas urbanas en proceso de degradación. En el establecimiento de estos convenios con los entes públicos de los diversos bilitación, la gestión y reparto de las ayudas, y el control de las actuaciones que dependen del mismo. Estos convenios recogen las actuaciones a las que se comprometen los entes públicos, los fondos que aportarían cada uno de ellos y el número mínimo de intervenciones a realizar en el período para el cual se firma el acuerdo. No obstante, también se ha de señalar que en los últimos tiempos se están llevando a cabo ciertas prórrogas para permitir que los municipios lleguen a alcanzar los objetivos a los que se habían comprometido. En este sentido, la Xunta habría firmado uno de estos convenios en el año 2002, estableciéndose en el Decreto 199/2002, de 6 de junio, las ayudas públicas a las cuales se podría acceder para la rehabilitación, así como la gestión de las previstas por el Estado en el Real Decreto 1/2002, para el período 2002-2005.

Mapa 1 Áreas de Rehabilitación en Galicia, 2007

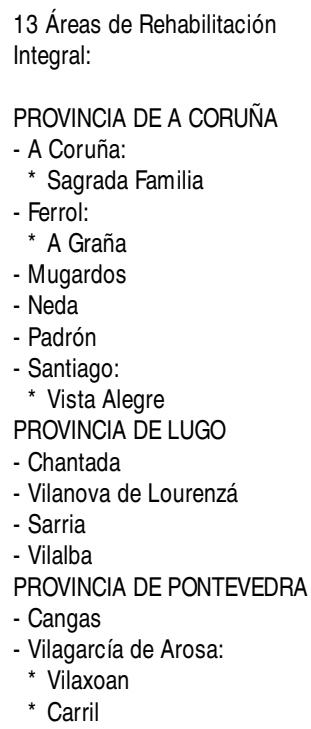

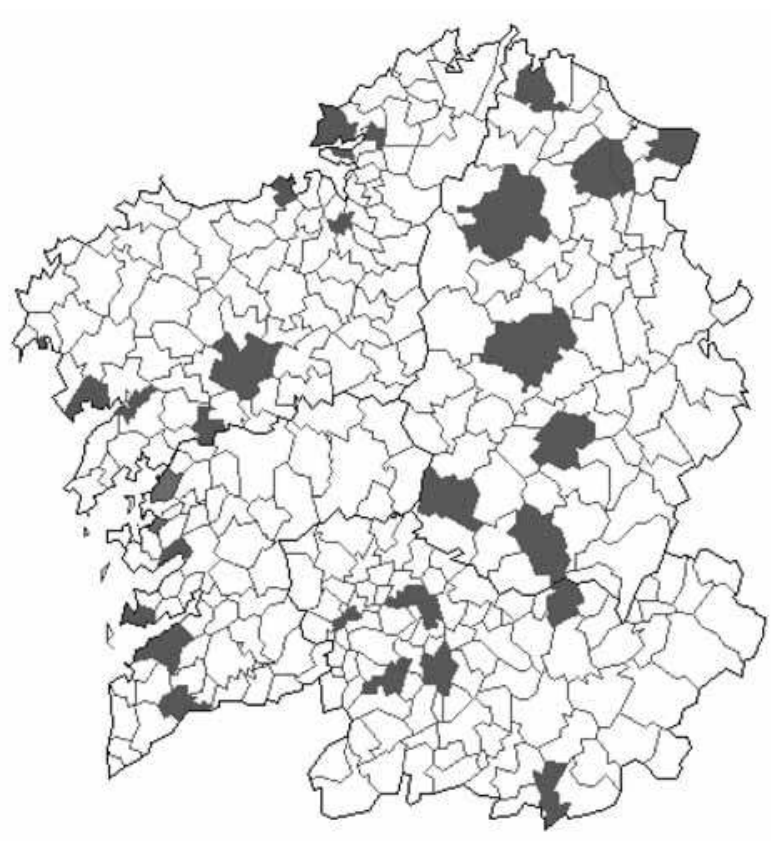

24 Centros Históricos:

PROVINCIA DE A CORUÑA

- Betanzos

- Corcubión

- Muros

- Noia

- Santiago de Compostela

- A Coruña:

* Ciudad Vieja

- Ferrol:

* Ferrol Vello

* A Magdalena

PROVINCIA DE LUGO

- Lugo

- Mondoñedo

- Monforte de Lemos

- Ribbadeo

- Viveiro

PROVINCIA DE OURENSE

- Allariz

- Castro Caldelas

- Celanova

- Ourense

- Ourense-Seixalbo

- Ribadavia

- Verín

PROVINCIA DE PONTEVEDRA

- Cambados

- Combarro-Poio

- Tui

- Vigo

Fuente: Ministerio de Vivienda y elaboración propia ${ }^{2}$

niveles implicados (estatal y local) intervendría el IGVS, como organismo autónomo que tendría entre sus funciones la promoción y gestión de los planes y programas en materia de vivienda y suelo; por tanto, las actuaciones relativas a la rehabilitación de viviendas, el reconocimiento de las áreas de reha-

\section{Metodología}

Para el desarrollo de nuestra investigación, acerca de la importancia del capital social en la promoción y desarrollo de las políticas de revitalización de zonas urbanas en declive, nos planteamos 
la utilización de una metodología marcada por la combinación de técnicas cualitativas y cuantitativas, puesto que ambos tipos de técnicas poseen unas potencialidades y deficiencias propias susceptibles de ser aprovechadas o superadas para obtener una visión más global de la situación del objeto de nuestro análisis.

Si bien el análisis de los datos secundarios nos puede aportar una radiografía del "estado de la cuestión”, una visión macro, para localizar y explicitar lo que podríamos denominar los síntomas del declive socioeconómico de una determinada zona, este declive afecta a una gran diversidad de personas, con concepciones e intereses que no tienen necesariamente que coincidir con las de los especialistas. Resulta por tanto necesario humanizar esos datos, esto es, combinar la visión macro con una visión micro, fruto de la aplicación de técnicas cualitativas para llegar a conocer en mayor profundidad, no sólo cómo se manifiesta ese declive, sino también cómo lo interpretan e intentan hacerle frente las personas más directamente afectadas por el mismo.

Teniendo cuenta de las presentes matizaciones, se decidió la aplicación del análisis de los documentos y datos secundarios derivados de la puesta en marcha del plan de rehabilitación en los barrios de Ferrol Vello y La Magdalena, esto es, el diagnóstico de la situación en estos barrios realizado antes de la puesta en marcha del plan (materializado en la Memoria del área de rehabilitación para los barrios históricos de Ferrol Vello-Magdalena, Ferrol), así como los datos sociodemográficos derivados de las fichas realizadas por la Oficina de Rehabilitación (Ayuntamiento de Ferrol) y otros registros. Mas la información que se ha podido extraer de los referidos documentos, por cuestiones como el diseño para recoger información de tipo arquitectónico más que para la de tipo social, han presentado múltiples problemas a la hora de abordar su análisis; en parte por la multiplicidad de valores perdidos en diversas casillas de las fichas y escasez de datos sociodemográficos sobre las personas que participan en el Plan. Estos problemas iniciales serían afrontados mediante el análisis de datos secundarios provenientes de fuentes oficiales que, especialmente por la explotación del Censo de Población de 2001, permitieron llevar a cabo un cruce de informaciones a fin de poder bosquejar una imagen más nítida sobre la forma en que esta política social está influyendo sobre el ámbito de estudio elegido.

A fin de profundizar un poco más en los datos obtenidos y ampliar su análisis, se han realizado entrevistas a tres participantes en el plan de rehabilitación, al director de la Oficina de Rehabilitación y a una experta en patrimonio que, además de haber nacido y vivido siempre en uno de los barrios, está realizando labores similares sobre rehabilitación de zonas históricas en una importante ciudad de Portu- gal. Fuentes de información todas éstas que hemos ampliado a través de la confección de un dossier de prensa con las noticias ferrolanas, recogidas de un importante periódico de Galicia ( La Voz de Galicia), sobre temas como el propio Plan de Rehabilitación y otras noticias relacionadas.

Esta misma multiplicidad de informaciones extraídas a distintos niveles, para dar lugar a un análisis combinado, ha hecho que fuera necesaria la adopción de unas estrategias específicas por la falta de uniformidad en las zonas que consideran los diversos agentes encargados de producir la información. Destacando principalmente las notables diferencias existentes entre las zonas contempladas por el Censo con respecto al informe acerca de las áreas de rehabilitación, pues la determinación de las secciones censales derivan de decisiones administrativas que no siempre respetan las fuertes relaciones que se dan, tanto por temas históricos como de homogeneidad de características, dentro de determinadas zonas, como ejemplifica el caso de que se separe uno de los márgenes de una calle de un mismo barrio para incluirlo en otra sección censal con características notablemente diferentes. Por tanto, se han tenido que forzar los datos para intentar llevar a cabo nuestras estimaciones e incluir zonas que decididamente no corresponderían a los barrios de nuestro interés.

\section{El Plan de Rehabilitación de Ferrol Vello y la Magdalena}

Nacida como una típica villa marinera gallega, Ferrol constituye, con sus aproximadam ente 77.000 habitantes (INE 2005), la sexta ciudad gallega en importancia. Su configuración urbanística, fruto de una evolución histórica marcada por su fuerte dependencia con respecto al sector naval y sus etapas de auge y declive, cuenta con diversos barrios que pueden ser tomados como notables ejemplos de su época. En este sentido, se pueden observar en su plano las características de los asentamientos medievales, con sus calles estrechas e irregulares, en el barrio de Ferrol Vello; el urbanismo racionalista del siglo XVIII, fruto del desarrollo de los planes por parte de ingenieros o arquitectos militares, en el barrio de la Magdalena; e incluso, las características casas baratas erigidas durante el período desarrollista del franquismo para dar alojamiento a la afluencia de mano de obra que acudía a la ciudad, como es el caso del barrio de Recimil, alrededor del cual se ha establecido el mismo debate sobre las opciones de renovación o rehabilitación de las cuales se ha hablado anteriormente.

Los orígenes del Plan de Rehabilitación se pueden remontar más o menos al año 2000, cando arquitectos relacionados con el Ayuntamiento llevarían a cabo una investigación acerca del estado del 
parque de viviendas en los dos barrios históricos mencionados. Esa investigación, que no podría llegar a alcanzar cubrir la totalidad de dicho parque de viviendas (bien por negativa de acceso de los propietarios a las viviendas o por hallarse estas vacías), tuvo como resultado la redacción de una memoria que arrojaba unos resultados bastantes nefastos en cuanto a la situación de ambos barrios. por medio de la gráfica de la siguiente página, que presenta el tipo de vivienda familiar en relación al estado del edificio en que se encuentran.

Valores que contrastan de forma clara con los valores medios para el total del municipio de Ferrol, pues de las 39.148 viviendas que existían en total en el año 2001, según el Censo de población y viviendas, únicamente un $2,88 \%$ se encontraban en

Mapa $2 \triangleright$ Delimitación espacial de las Áreas de Rehabilitación Integral de Ferrol

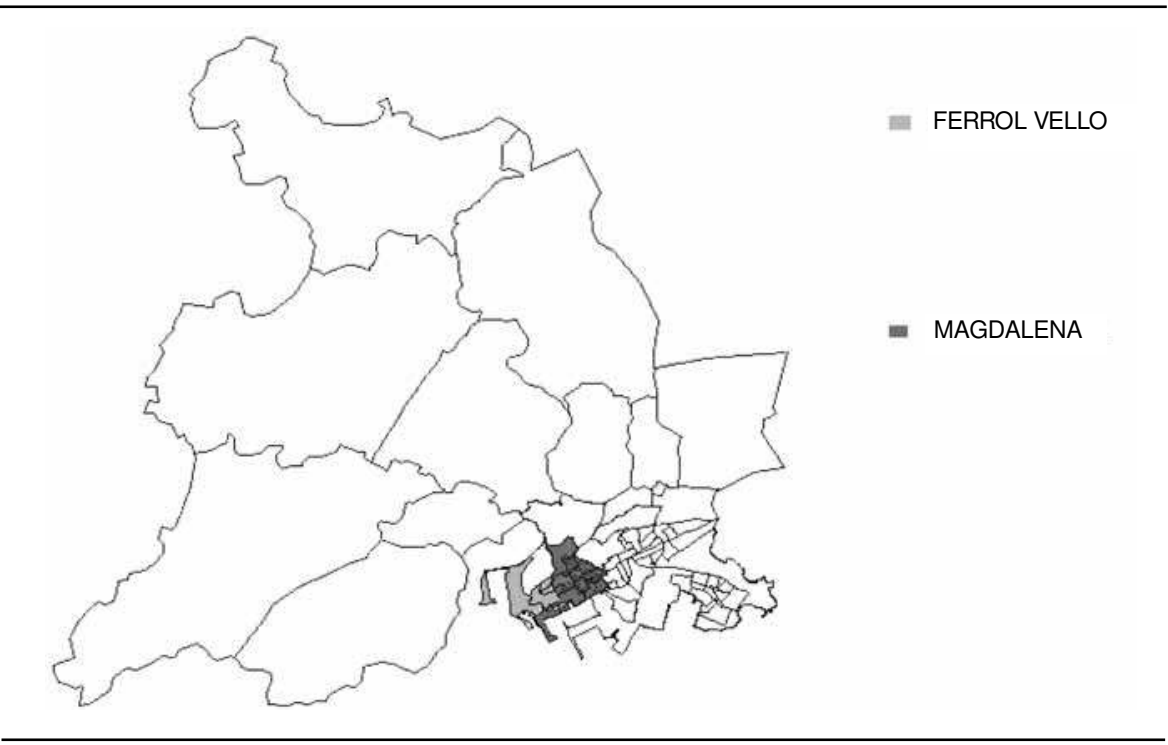

Fuente: INE, Censo de Población, 2001 y elaboración propia

La situación del parque de viviendas en el barrio de Ferrol Vello resulta bastante preocupante, debido al proceso de degradación a que se han visto abocadas por el abandono del mismo, como refleja que el $43,3 \%$ de las viviendas estudiadas (489 en un total de 159 edificios) se encontraran deshabitadas en el momento del estudio, pero estando solamente un $10,2 \%$ de ellas en disponibilidad de ser vendidas o alquiladas. De media, si se analizan las deficiencias, alrededor de la mitad de los edificios, con niveles que varían entre el 40 y el $60 \%$ según los casos, presentan problemas con sus infraestructuras básicas (revestimientos, instalaciones eléctricas, etc.), lo que daría lugar a que un $51,6 \%$ de edificios considerados como en mal o regular estado.

Con respecto al barrio de la Magdalena, según los diversos aspectos tomados en cuenta (fachadas, galerías, instalación eléctrica o estado general del edificio) por los arquitectos, se alcanzaban porcentajes de entre un 10 y un $20 \%$ de los edificios/viviendas revisados calificados como en mal estado y un $50 \%$ calificado como en estado regular. Todo lo cual queda más claro si se analiza la totalidad de las dos áreas mal estado y un $14,74 \%$ en estado deficiente ( 820 y 4.204 viviendas, respectivamente), sobre todo si se tiene en cuenta que no sería el total de viviendas existentes en las dos áreas de rehabilitación las que se han revisado, sino únicamente una parte de ellas a las que se ha permitido el acceso.

Como se puede observar, parece que podemos constatar la existencia de una notable correlación entre el abandono de las viviendas y su deterioro por falta de mantenimiento, lo cual resulta necesario para su habitabilidad. Por un lado, el mayor porcentaje de las viviendas situadas en edificios en estado ruinoso se concentran en aquellas que se encuentran vacías, mientras que, por el otro, el mayor porcentaje de las viviendas familiares en buen estado se concentran en aquellas calificadas como viviendas familiares principales. Cuestión esta última que nos hace plantearnos por qué se da ese abandono de las construcciones hasta su ruina. ¿Como una forma de favorecer posteriores acciones de tipo especulativo con el suelo sobre el que están asentados los edificios?, ¿por incapacidad de hacer frente a los costos de su mantenimiento? 
Gráfico 1 Tipo de viviendas familiares por estado del edificio, áreas de rehabilitación de Ferrol Vello y la Magdalena ${ }^{3}$

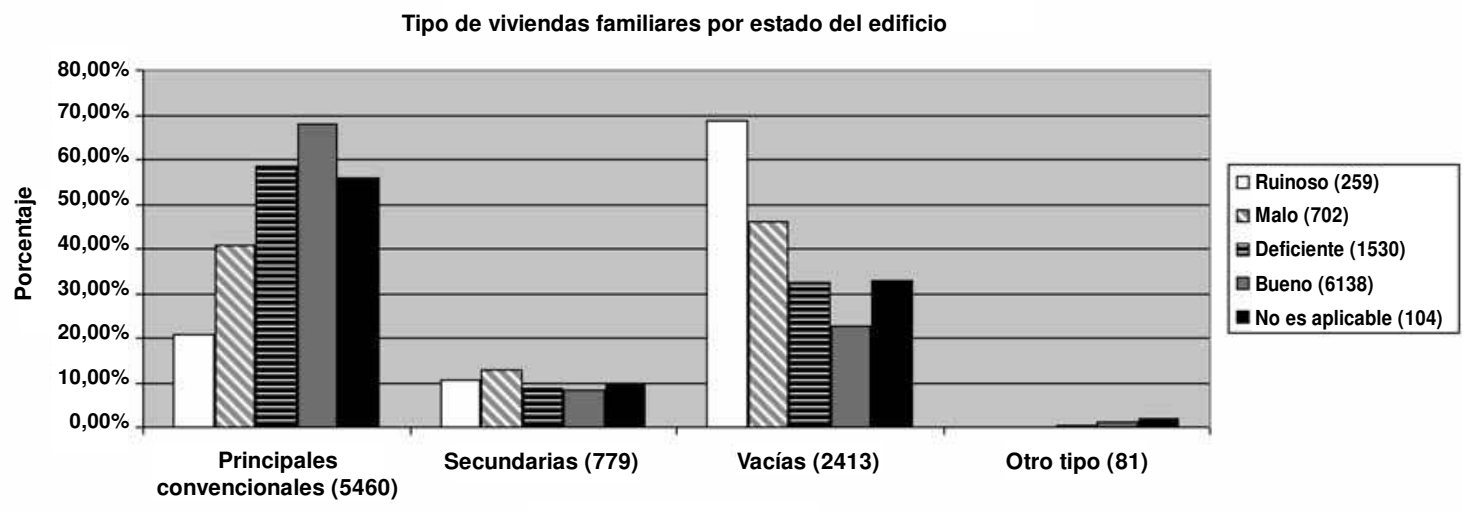

Tipo de viviendas familiares

Fuente: INE, Censo de Población y viviendas, 2001 y elaboración propia

o ¿por mera indiferencia a lo que le está ocurriendo a este patrimonio arquitectónico?

El abandono en que se encuentran sometidos los centros históricos en gran parte de Galicia, a pesar de las relativam ente recientes intervenciones que se están poniendo en práctica en ciudades como Lugo, La Coruña o Vigo, resulta claramente visible si se realizan visitas a estos lugares o en noticias de prensa que hacen referencia a los mismos, como es el caso de la noticia publicada en La Voz de Galicia bajo el título "Chequeo a los centros históricos" (23 de febrero de 2006) señalando la mala situación de varios centros históricos en grandes ciudades de Galicia y lo caro del precio del metro cuadrado en algún de ellos.

Para hacer frente a este tipo de patologías se desarrollan las medidas establecidas por los planes de rehabilitación, no sólo para mejorar la calidad de vida de los ya residentes y fijarlos en el espacio, sino también para atraer nueva población que pueda darle vida a la zona y sacar partido a unos inmuebles que, ya sea por abandono o mal mantenimiento, no son considerados como adecuados para la vida en esos barrios concretos, reforzando así la dinámica de abandono y degradación del entorno.

El primer paso para la materialización de las medidas a adoptar lo constituyó la creación de la Oficina de Rehabilitación el 7 de abril de 2002, tras la firma del contrato con los arquitectos que se pusieron al frente de la misma y la cumplimentación de los trámites burocráticos que permitirían el comienzo de las actividades en el área, iniciándose la recepción de solicitudes a partir del verano de ese año. A este respecto, destaca el hecho de que alrededor de una décima parte de las solicitudes recibidas en los casi cuatro años de andadura del plan analizado (un total de 134 de las 1331 con- tabilizadas a la fecha de terminación del estudio) fueran realizadas en un solo día, el 6 de agosto de ese mismo año.

Dichas subvenciones consistirían en dos tipos básicamente, además de los créditos con intereses blandos negociados por el Ayuntamiento con entidades bancarias, las subvenciones por área, a las que podrían optar todos los edificios existentes en las áreas de rehabilitación y cuyo porcentaje aplicable sobre el coste de la rehabilitación variaría entre el 25 y el $55 \%$, en función del elemento que entra en el Plan de Rehabilitación (es decir, su catalogación, que establecería también unas cuantías máximas) $y$ del nivel de ingresos del solicitante. Por otra parte, cuentan también con la posibilidad de optar a una subvención por conjunto histórico los edificios situados en el barrio de la Magdalena, al haber sido declarado conjunto histórico, que permitiría ampliar el porcentaje de subvención hasta el $75 \%$ del límite máximo establecido según el nivel de catalogación del edificio.

Pero los recursos dedicados a esta política resultan ciertamente escasos y las oficinas de rehabilitación han de realizar notables cribas en el reparto de los mismos. El planteamiento seguido para la marcha de dicho plan se basaría en la generación de una inquietud de rehabilitación, en palabras del director de la oficina, por "contagio" 4 . Éste se basaría en la premisa de que la Oficina no está ahí para la rehabilitación de todos y cada uno de los edificios, sino más bien para generar en la ciudadanía una cultura de la rehabilitación que los lleve a emprender e invertir en sus propios proyectos, con lo que les interesan especialmente lo que denominan proyectos "externos", esto es, llevados a cabo por profesionales no relacionados con el ayuntamiento a petición de los propietarios de los inmuebles. 
Esa generación del contagio cuenta con la colaboración de diversas agencias y agentes, situación que podemos asimilar a la planteada por el tema de la educación, toda vez que también implica una cierta socialización de la población y su concienciación acerca de una cuestión de notable importancia, como serían los medios de comunicación, los grupos de pares (amigos o conocidos), los políticos, etc., tema éste que abordaremos en mayor amplitud en el siguiente epígrafe. Lo que se pretende favorecer también con la dispersión de las actuaciones por diversas zonas de las áreas de rehabilitación, de tal forma que éstas pudieran ser vistas en la mayor parte del territorio afectado por esta medida, para que la observación directa de los propios resultados que se van obteniendo con la aplicación de este tipo de medidas, a modo de efecto demostración, coadyuve en esa generación de la inquietud de rehabilitar.

Expresión de esa consecución de objetivos por parte de la actividad de la Oficina de Rehabilitación del Ayuntamiento de Ferrol se vería reflejada en nuestra estimación de la inquietud por rehabilitar, fruto del cruce de los datos aportados por la Memoria del área de rehabilitación para los barrios históricos de Ferrol Vello-Magdalena con los del Censo de Población y Viviendas de 2001. Hablamos de un cierto éxito del plan haciendo referencia a que se ha conseguido fom entar la rehabilitación del patrimonio arquitectónico más allá de los límites que supone la escasez de medios con que suele contar la administración, es decir, que muchos más propietarios que los que reciben ayudas para rehabilitar pongan en marcha sus propios proyectos.

Así, como se verá en la tabla situada bajo estas líneas, aunque no se hayan alcanzado grandes porcentajes de edificios/viviendas rehabilitados sobre el total del parque de viviendas existente, tanto el número de solicitudes realizadas como la escasez de las subvenciones concedidas (sólo 242 subvenciones - 83 subvenciones por área de rehabilitación y 159 por conjuntos históricos - para un total de 1331 solicitudes), junto con los diversos problemas de tipo metodológico a la hora de analizar y valorar los resultados a los que se ha hecho referencia en el apartado de la metodología, nos permiten ser bastante optimistas en cuanto a la marcha del plan de rehabilitación. En este sentido, suponemos que si contáramos con datos más precisos y recientes sobre las zonas estudiadas, esto es, que abarcaran únicamente estas dos áreas de rehabilitación y con fecha más próxima que la de realización del censo, los porcentajes estimados habrían de resulta sensiblemente superiores por haberse eliminado uno de los grandes escollos a la investigación sobre el tema de la vivienda en entornos urbanos.

Los problemas metodológicos aludidos, relativos a la no completa coincidencia entre las secciones censales tomadas en cuenta para el análisis del área de rehabilitación y el área comprendida por esta última, derivados de la forma en que son definidas administrativamente las secciones censales, han provocado que se tuviese que adoptar un enfoque bastante riguroso para la delimitación de las áreas de rehabilitación, obligándonos a considerar una sección censal que contenía únicamente un margen de una calle donde se estaban llevando a cabo obras de rehabilitación, pero que, además, contemplaba otras calles que estaban fuera de dicha área y que contaban con edificaciones en altura de cierta entidad, con lo que ejercen un notable efecto de disminución de los porcentajes estimados de participación por tratarse de términos relativos.

Así, a tenor de los datos desnudos, en principio la inquietud por rehabilitar no estaría muy extendida, pero poniéndolos en relación con su contexto, a través de las informaciones derivadas tanto de la memoria realizada por la Oficina de Planeamiento como del conocimiento de que no todos los que están llevando a cabo procesos de rehabilitación estarían incluidos dentro del Plan, sino que realizarían dichas gestiones por su cuenta, el panorama cambiaría relativamente. Inicialmente habría en Ferrol Vello un $17,3 \%$ de las viviendas revisadas por los técnicos (revisadas y no el total) que se encontraban en malas condiciones, mientras que el porcentaje estimado de solicitudes de intervención sería del 6,91\% sobre el total de viviendas, con lo que parece que sí existe un cierto éxito por parte del Plan en dicha zona, al estimar que gran parte de esas intervenciones actuaría precisamente sobre las que presentan deficiencias. Lo que se repite para el caso del barrio de la Mag-

Tabla $1 \triangleright$ Estimación de la extensión de la inquietud por rehabilitar en las áreas de rehabilitación de Ferrol Vello y la Magdalena

\begin{tabular}{|l|c|c|c|}
\hline & Total solicitudes & Total viviendas & $\begin{array}{c}\text { \% solicitudes } \\
\text { sobre total }\end{array}$ \\
\hline Total viviendas & 663 & 8733 & 7,59 \\
\hline Barrio Magdalena & 502 & 6404 & 7,84 \\
\hline Barrio Ferrol Vello & 161 & 2329 & 6,91 \\
\hline
\end{tabular}

Fuente: INE, Censo de Población y Viviendas, 2001; Fichas solicitudes Oficina Rehabilitación de Ferrol, 2002-2006 y elaboración propia 
dalena aunque con menos fuerza, pues, según la Memoria, sería un $30 \%$ el porcentaje de viviendas que se encontraban en mal estado y se solicitaría la intervención para el $7,84 \%$ de las viviendas existentes. No podemos dejar de destacar esta aparente mayor implicación en el caso de Ferrol Vello, lo que más adelante quizá pueda ser explicado en parte por nuestro análisis de la influencia del capital social en la participación ciudadana.

\section{Capital social y participación ciudadana en la rehabilitación del casco histórico de Ferrol}

El tema del capital social ha sido abordado históricamente desde perspectivas similares, aunque haciendo hincapié en aspectos más o menos amplios de este concepto. Todas ellas hacen referencia a la red de relaciones y normas que, ante la necesidad o deseo de alcanzar un determinado fin, sirven de apoyo para la consecución de unos determinados efectos. Sentido éste que se puede extraer, desde el inicio de su definición, por parte de Lyda Judson Hanifan $^{5}$ a principios del siglo pasado, cuando se aplica este concepto para hacer referencia a los elementos o aspectos que facilitarían la acción de los individuos hacia la consecución de determinados fines, más allá del tradicional énfasis en una mano invisible que haría que la persecución de los propios objetivos terminara por beneficiar a la comunidad, por la unión de fuerzas en una suerte de red social.

Este mismo enfoque lo podemos encontrar también, con ligeras variaciones, en la definición dada por James S. Coleman, para quien sería: “... una variedad de entidades diferentes que tienen dos características en común: todas ellas consisten en algún aspecto de una estructura social, y facilitan ciertas acciones de individuos que están dentro de la estructura." (Coleman, 1994: 302).

Encontramos ya, por tanto, una mención más explícita que en Hanifan de este papel facilitador de la acción que posee el capital social para la consecución de un objetivo determinado, la cual podemos ver abordada en mayor amplitud en diversas obras de Putnam, quien introduciría en su definición del concepto tres aspectos que resultan de notable importancia (al poder actuar en una suerte de circuito de retroalimentación): “... los aspectos de la organización social tales como la confianza, normas y redes, que pueden mejorar la eficiencia de una sociedad al facilitar la acción coordinada." (Putnam cit in Millán e Gordon, 2004: 725).

Siendo este sentido de elemento facilitador de la acción coordinada para la consecución de un objetivo dado el que más nos interesa en nuestro análisis de los factores que entrarían en juego para alcanzar lo que definimos como un cierto éxito del plan de rehabilitación, pues consideramos que la existencia de un fuerte capital social en el caso de la ciudad de Ferrol ha hecho mucho más fácil la extensión de la inquietud por rehabilitar entre la ciudadanía. Esto es, la formación y aprovechamiento de las redes sociales tendrían una notable influencia en la participación ciudadana en el desarrollo de un plan de rehabilitación en los barrios ferrolanos de la Magdalena y Ferrol Vello, pues aportarían una serie de efectos positivos, posibilitando una mejora en la transferencia de informaciones, que fomentarían de forma directa o indirecta la implicación de la población residente para el logro de recuperar y revitalizar sus barrios.

Así, teniendo en cuenta que una de las críticas más comunes en relación a la puesta en práctica de diversas políticas sociales sería la escasa disposición de informaciones o que éstas no llegarían al total de la población, estas redes facilitarían que éstas llegaran de forma más rápida y sencilla a un mayor número de ciudadanos por haberles llegado por diversos canales, a saber, a través de medios de comunicación, por comentarios de amigos y/o conocidos, por ver directamente los resultados de intervenciones sobre edificios concretos y contar éstos con carteles anunciadores de esta actividad, etc.

Ejemplificador de este papel transmisor de información del capital social resulta el testimonio de una de las personas que habían realizado la rehabilitación de un edificio de su propiedad, cuando comenta cómo un amigo suyo se enteraría a través de él de la existencia del Plan y las ayudas a las que podría optar para poner en marcha un proyecto de rehabilitación propio.

Sí, sí, sí. Aún el otro día...el otro día, me encontré con un amigo...de hace muchos años ya, de 20 años o así, y me dice "oye, arreglaste la casa y...y lo arreglaste por..." "Sí" Y me dijo, "oye, cómo es eso" "Pues es así y tal, y tal, y tal" Y me dijo "¿Y nada más? Yo tengo que ir aquí y ellos me miran todo y miran todos los planes y tal" "Sí. Tú tendrás tu idea, claro, cómo te gustaría que fuera y tal" Y me dijo "Pues resulta que tengo una casa en la calle María y oye, o la arreglo o se me cae" Y dije yo "Pues vete por allí..." (Hombre de 72 años residente en el barrio de la Magdalena)

De lo expuesto se podría pensar que los que llevan a cabo la rehabilitación simplemente pretenden beneficiarse ellos mismos de las ayudas que se conceden para tal fin y que sería la suma de esos esfuerzos personales en la búsqueda de su propio beneficio la que, al igual que el planteamiento de la mano invisible del mercado, conllevaría una mejora en el entorno constituido por estos barrios. Pero 
encontramos una serie de cuestiones que ponen en entredicho dicho enfoque: por un lado, si yo rehabilito pero tú no, ¿la continuación de la degradación del entorno no me afectará igualmente a mí a pesar de mis esfuerzos al respecto?; por el otro, la aparición de un movimiento social que pretende impulsar la rehabilitación e incluso la implicación para la mejora del barrio de Ferrol Vello, ya sea actuando directamente en algunas de las situaciones en las cuales el ayuntamiento ha presentado una cierta dejación de sus funciones con respecto a la labor de desescombro por una casa que se había derruido por un incendio ${ }^{6}$ o bien presentando ideas para dicho entorno.

Este sería el caso de la constitución de la denominada Plataforma de Defensa de Ferrol Vello, la cual sería explicada por el arquitecto de la Oficina como consecuencia de la radicación en el barrio de recién llegados más alternativos con respecto a la población de siempre, los cuales entenderían de otra manera la calidad de vida, facilitando en cierta medida la intervención, de tal manera que la degradación daría paso a un espacio muy deseable, al mismo tiempo que la especulación que se sabe que ya existe se encuentra con el control de las acciones llevadas a cabo por esta misma plataforma vecinal. llevar a cabo intervenciones, etc. Profesionales de diversos cam pos dejan su impronta en la actividad de la asociación constituida para mejorar ese entorno, aportando no sólo visiones críticas acerca de lo que se está llevando a cabo en el lugar, sino mostrando alternativas de lo que podría ser llevado a cabo, surgiendo líderes que fomentan la implicación de los vecinos en la suerte que puede correr su barrio. Mas, al mismo tiempo, esta misma asociación vecinal establece nuevos nexos con otras asociaciones del entorno para una mayor difusión, apoyo popular y coordinación en los actos reivindicativos, como sería la integración del Comité Cidadá pola Recuperación de Ferrol Vello (o plataforma, pues se pueden encontrar documentos con diversas denominaciones) dentro de la Plataforma Ártabra $21^{7}$, creada en 2006 y entre cuyos fines se encuentra el favorecer la participación ciudadana.

Como ya señalara Putnam, con respecto al surgimiento de líderes, éstos pueden llevar, como hemos comprobado, a la generación de una mayor implicación o un mayor movimiento, en el sentido de generar una actitud más reivindicativa de lo que constituyen sus derechos. Así, este barrio contaría en la actualidad con un movimiento ciudadano bastante organizado, activo y reivindicativo: llegando a man-

Gráfico $2 \triangleright$ Comparativa población del barrio de Ferrol Vello, por grupos quinquenales de edad, 2001-2006

Población del Ferrol Vello por grupos quinquenales de edad, 2001-2006

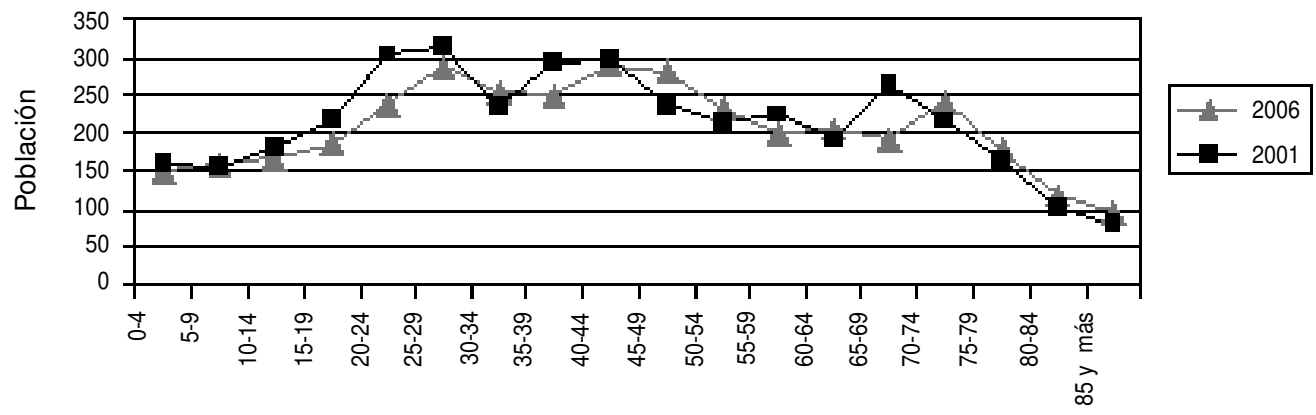

Grupo de edad

Fuente: INE, Censo de Población y Viviendas, 2001; INE, Padrón Municipal de Habitantes, 2006

El barrio de Ferrol Vello ha seguido sufriendo pérdidas de población en los últimos años, sin embargo, se ha registrado un cierto incremento de la población en determinados grupos de edad que, a pesar de no ser joven (se sitúan los mayores aumentos entre la población de 45-49 años), enriquecen las posibles visiones acerca de lo que desean para su barrio y su entorno, cómo se podrían tener reuniones con la Dirección de Patrimonio de la Xunta de Galicia para presentar propuestas y que ha sido capaz de organizar una partida de limpieza para retirar escombros. Con lo que parece ser que la fuerza de este movimiento ciudadano está en parte basada en la llegada de nuevos habitantes a la zona, entre los que se contarían varios arquitectos (con las posibilidades que se abren en cuanto a la capacidad 
de presentar alternativas, generar debate, aportar nuevas visiones, etc., que esto puede suponer), dinamizando la acción de los habitantes de esta zona para proteger el patrimonio arquitectónico que posee la zona, organizándose frente a los desmanes de Ayuntamiento y particulares, etc.

Todo lo cual parece continuar con la fuerte tradición de movimientos obreros y asociacionismo hondamente calada en la evolución histórica de la ciudad desde la ubicación de unos astilleros dedicados especialmente, desde mediados del siglo XVIII, a la construcción de buques de guerra y la consecuente constitución de un creciente proletariado urbano. Ejemplo de este hecho sería la organización de los obreros, a pesar de la represión de la que eran objeto en plena época franquista, para hacer frente a diversos intentos de despido, a los convenios colectivos del sector, etc., mediante la convocatoria de manifestaciones y huelgas en las que destacaría la solidaridad ciudadana con el mismo. La manifestación popular con mayores consecuencias, en la que morirían dos obreros, sería la acaecida en marzo de 1972, en un ambiente de fuerte conflictividad política y laboral. En esta época estaban siendo realizados los convenios colectivos del sector, pero el enfrentamiento de los trabajadores (que solicitaban un convenio a nivel de la factoría) con la dirección de la empresa, el Sindicato Vertical y las autoridades gubernamentales, provocaría medidas de presión para lograr sus propósitos. Tras la firma en Madrid del V Convenio y haber sido objeto de sanciones diversos enlaces sindicales de CC.OO., los trabajadores se concentraron delante de la puerta de Bazán, marchando en manifestación para la lograr la solidaridad de los empleados de las otras empresas. Pero, en las inmediaciones del Puente das Pías, éstos son interceptados por las fuerzas del orden, que utilizan sus armas contra los trabajadores, dejando un saldo de dos muertos y más de 40 heridos.

Mas no se vería únicamente una organización como movimiento obrero reivindicatorio, sino que resulta también destacable el espíritu asociativo en la ciudad, en cuanto éste sería quizá la expresión más visible del capital social existente en la misma, materializado en la constitución de fundaciones con objetivos socioeconómicos, como sería la Fundación Ferrol Metropolitano (1998), la cual, con la colaboración de empresas, instituciones locales y la administración, comenzaría a promover varios proyectos para la revitalización económica de la zona: creación de un Centro Comercial Abierto en el barrio de la Magdalena, impulso del Foro de Ciudades de la llustración, etc. Así como la constitución de asociaciones de vecinos en relación a la materialización de sus derechos, como un movimiento de reivindicación de, y actuación sobre, los problemas que presenta la configuración urbanística de los barrios.
El propio enfoque adoptado para la puesta en marcha y desarrollo del Plan de Rehabilitación, centrado más en la generación de proyectos propios de rehabilitación por parte de los propietarios de inmuebles en dichas áreas de rehabilitación que en la mera concesión de subvenciones, encuentros con los vecinos o el establecimiento de acuerdos con empresas constructoras y entidades bancarias, pone de relieve la importancia del capital social para la participación de los ciudadanos en el mismo. La persecución del objetivo de recuperar los edificios y las viviendas, así como del entorno de los barrios y de población, a través de una estrategia que pretende impulsar la iniciativa privada, dado el bajo número de subvenciones que son concedidas, orienta nuestro análisis en esa dirección. Sin embargo, a pesar de que el propio director de la Oficina señalara que no se había realizado demasiada publicidad del plan, el dossier de prensa, extraído de las informaciones ofrecidas por La Voz de Galicia en los últimos seis años, que hemos reunido parece señalar precisamente lo contrario, toda vez que la mayoría de las noticias derivarían de la acción publicitaria del Ayuntamiento.

Porque no sería solamente desde el Ayuntamiento, y las oficinas de la Administración relacionadas con el mismo, de donde deriva la información y el apoyo a la extensión de la cultura de la rehabilitación en estas zonas de la ciudad, sino que se verían implicadas además otras agencias y agentes:

1) El papel de los medios de comunicación como agente de socialización y concienciación sobre la necesidad de rehabilitar haciendo un seguimiento de las actividades de rehabilitación, publicitando la apertura de períodos de solicitud de subvenciones, entrevistando a profesionales que aportan sus visiones al respecto, etc. Lo que se comprobaría al observar la multitud de informaciones que se traducirían en un dossier de más de 100 páginas, sobre todo si se tiene en cuenta que éste correspondería únicamente a uno de los periódicos que pueden ser comprados en la zona.

2) La firma de acuerdos del Ayuntamiento con entidades bancarias para la tramitación de créditos con intereses blandos, permitiendo así a la población medios que les habrían de hacer más llevaderos los pagos de los costes de unas actuaciones que no resultan nada baratas y que, en caso contrario, podrían ciertamente impedir la puesta en marcha de las mismas, con el consiguiente menoscabo para la recuperación de las zonas.

3) Por otra parte, el Ayuntamiento también negociaría con empresas de construcción el establecimiento de unos precios más o menos estándar para este tipo de 
actuaciones, facilitando no sólo el coste sino también el control de los resultados, sobre todo tras la constitución de una homologación para empresas que puedan Ilevarlas a cabo. De esta manera, no sólo las empresas encuentran un floreciente mercado para sus actividades, dándose el caso de que algunos interesados en la rehabilitación de los edificios o viviendas han de esperar algún tiempo para ponerlas en marcha por la demanda existente, sino que éstos últimos cuentan con unas ciertas garantías en cuanto a la calidad del trabajo y el Ayuntamiento de que se respetan los límites establecidos por las ordenanzas pertinentes.

4) La acción coordinada de diversas asociaciones vecinales a la hora de exponer los problemas que afectan a sus barrios, la publicidad de posibles alternativas que pueden ser desarrolladas por los poderes públicos y el contacto con los mismos a través de los canales formales, así como la utilización de las nuevas tecnologías de la información para facilitar el acceso a sus propuestas y acciones además poder recoger nuevas visiones que generen debate. Pero también la denuncia de las desigualdades de unas zonas con respecto a otras, tanto en términos de acciones como de inversiones en las mismas, favoreciendo el deterioro y posibles especulaciones en un futuro más o menos próximo. En resumen, la facilitación de la participación ciudadana más allá de la mera acción de votar a un determinado partido, como forma de apoyo a y de control de la actividad de los entes públicos.

5) Por último, en los edificios en que son varios los propietarios es necesario llegar a un acuerdo entre todos los vecinos, lo que no suele ser fácil muchas veces, de tal forma que actúen de forma relativamente solidaria entre ellos, lo que constituye además unos de los requisitos solicitados por la Oficina de Rehabilitación.

Teniendo en cuenta esta variedad de actores en la marcha del plan, siguiendo lo trazado con los enfoques de Putnam y Coleman, podemos señalar que no se trata simplemente de una egoísta persecución de beneficios personales, sino que existe una dialéctica de beneficios para la comunidad-beneficios personales que se retroalimenta. De nada serviría que se llevara a cabo la rehabilitación de una o unas pocas casas en un barrio tan degradado como estaría Ferrol Vello, por ejemplo, si esa mejora de las condiciones de vida en el propio hogar no se ve acompañada por una mejora del hábitat donde se encuentran ubicadas. El beneficio económico que se podría extraer del arrendamiento no estaría muy asegurado, al ser el estado o ambiente del barrio una de las condiciones que se suelen observar al buscar una vivienda para comprarla o arrendarla. Sería la implicación del mayor número posible de personas en la materialización de esos proyectos lo que reportaría los mayores beneficios, tanto para el propio individuo como para la comunidad en su conjunto; lo que no quiere decir necesariamente que el individuo actúe conscientemente como el actor de la teoría de la acción racional, como también señalaría Coleman.

En conclusión, las propias condiciones y el marco organizativo en que se está desarrollando el Plan de Rehabilitación hacen que la participación ciudadana en el mismo y el signo positivo de su marcha no sólo se apoye en el capital social, sino que incluso fomente la generación de mayores desarrollos del mismo, ya sea a través del surgimiento de nuevos líderes como por fomentar los nuevos movimientos sociales organizados de ciudadanos que han sido concienciados de la importancia de su participación en el mismo para mejorar la calidad de vida en su entorno.

\section{Conclusiones}

Diversas son las conclusiones que podemos extraer de la realización del presente estudio, aunque la principal sería la notable importancia que ha tenido el capital social existente en la zona para el fom ento de la participación ciudadana en la materialización y buena marcha que parece estar llevando el Plan de Rehabilitación estudiado. De este capital social se derivarían funciones como la extensión de la información a un número cada vez mayor de ciudadanos o la socialización en la importancia de rehabilitar estos espacios tan degradados; al mismo tiempo que esta participación ciudadana propiciaría la emergencia de movimientos sociales organizados y claram ente activos que fomentarían la ampliación de ese capital social (como es el caso de la coordinación de los movimientos ciudadanos en una plataforma, entre otros). No obstante, se ha de tener en cuenta que no siempre este mayor capital social de determinados grupos resulta necesariamente bueno para el bien de la comunidad, al poder hacer todavía más patentes las desigualdades sociales en términos de participación en la toma de decisiones y en los resultados de la misma.

Esa toma de conciencia y mayor participación de la ciudadanía resultaría también un buen complemento de la acción del Ayuntamiento, a través de su actividad legisladora y de control, en la lucha contra el riesgo de especulación urbanística que amenaza todo proceso de rehabilitación, al permitir el ejercicio de un mayor control sobre ese mismo poder local para que cumpla y haga cumplir esas normas. La 
actitud de denuncia asumida por las asociaciones vecinales "ponen sobre el tapete" temas que de no haberse realizado este tipo de acciones quedarían escondidos a la opinión pública, provocando al menos una reacción, más pronto o más tarde y sea en el sentido que sea, por parte de los entes implicados en el tema.

En este sentido, la colaboración de los poderes públicos con el sector privado y los ciudadanos en el desarrollo de las estrategias de desarrollo local resulta, pues, un elemento ciertamente importante si se quiere que los proyectos lleguen a buen puerto. En el caso estudiado, la colaboración público-privado ha favorecido una cierta agilización de los procedimientos que ha implicado la puesta en marcha y funcionamiento de este proyecto. Sin embargo, la propia carga de trabajo a la que han de hacer frente tanto los arquitectos de la Oficina como las propias constructoras hacen que el proceso rehabilitador no pueda ir más rápido de lo que va, lo cual, si bien resulta junto a la lentitud burocrática una de las quejas más comunes de los que realizan o han realizado un proyecto de rehabilitación, puede ser tomado como un indicador del propio éxito que esta medida de desarrollo local está teniendo.

Hasta ahora parecen estar lográndose algunos avances en la mejora de los barrios históricos de Ferrol, sin embargo, se ha de tener en cuenta que la rehabilitación de los mismos es una actividad que requiere contar con el largo plazo, por el efecto que producen límites como la escasez de recursos materiales y humanos, en términos de subvenciones y personas cualificadas para llevar a cabo las acciones, el gran peso y tradicional lentitud de la burocracia, etc.

Se abre así un prometedor campo de investigación, y de colaboración entre especialistas en diversas materias, a la hora de valorar los impactos sociales que este tipo de planes pueden introducir en las ciudades. Como hemos señalado, la recogida de información de las personas que participan en el Plan de Rehabilitación sufre notables carencias con relación a la posibilidad de valorar esos impactos, lo que nos abre a los sociólogos urbanos un próspero campo de intervención dentro de los grupos multidisciplinares que sería deseable que participaran en el diseño y puesta en marcha de este tipo de proyectos, toda vez que, al fin y al cabo, lo que se busca precisamente es eso, provocar unos impactos sociales en los más diversos aspectos de la sociedad de la que forman parte, en la búsqueda de conseguir un desarrollo local que fomente realmente la calidad de vida de sus habitantes.

\section{Notas}

1 La Consellería de Ordenación do Territorio e Obras Públicas, a la cual se sumaría un organismo autónomo adscrito, el Instituto Galego de Vivenda e Solo (creado por la Ley $3 / 1988$ ), asumiría inicialmente las funciones relativas a vivienda. Sin embargo, la estructura organizativa del gobierno regional sufriría una reestructuración en fechas relativamente recientes cuando, al entrar en el gobierno regional el bipartito (PSG-PSOE y BNG), se separaron en dos consellerías distintas las competencias relativas a urbanismo y vivienda, englobadas inicialmente en la anterior. Esta separación, a parte de la falta de racionalidad que parecería mostrar el desglose de competencias de dos materias tan interrelacionadas, daría lugar a la creación de la Consellería de Vivenda e Solo como órgano independiente, mediante el Decreto 505/2005, del cual pasaría a depender desde entonces el IGVS.

2 Con la expresión elaboración propia hacemos referencia al tratamiento de los datos estadísticos realizados por los propios autores para presentar determinados análisis y que no se encuentran directamente disponibles en las fuentes estadísticas consultadas.

3 Entre paréntesis el número total de casos para cada una de las categorías de las variables contempladas

4 En este sentido, en más de una ocasión hemos tenido la oportunidad de escucharle hablar de este tipo de planteamiento al arquitecto e incluso una noticia referente a una entrevista con dicho director fue encabezada por el siguiente titular: "Rehabilitar es como un contagio"

5 En el sentido de "esos elementos tangibles [que] cuentan sumamente en la vida diaria de las personas, a saber, la buena voluntad, la camaradería, la comprensión y el trato social entre individuos y familias, características constitutivas de la unidad social" (Hanifan 1916: 130-138 cit in Castaño Martínez, 2005)

6 Noticia en prensa, "Los vecinos limpian la calle San Francisco de escombro", Diario de Ferrol, 8 de abril de 2006

7 A pesar de lo interesante que resulta el tema de la actividad de este tipo de asociaciones, la complejidad del entramado surgido en los últimos años en relación al movimiento vecinal hace que éste se escape en cierta medida del tema tratado en este artículo. No obstante, se cuenta con dos blogs que permiten conocer de forma bastante amplia las actividades de estas asociaciones, en los cuales, adoptando las nuevas tecnologías como una de las bases de facilitar el acceso a la información de las personas, se publicitan tanto los actos como los documentos derivados de sus iniciativas, se generan debates, etc. Sus direcciones son: http://artabra21. blogspot.com para la Plataforma Ártabra 21 y http:// www.jazztelia.com/ferrol-vello/blog para la asociación de Ferrol Vello.

\section{Bibliografía}

BOLETÍN OFICIAL DEL ESTADO, 1980-2006, varios números.

CASTAÑO MARTíNEZ, M. S. (2005), "Los valores éticos del capital social y su influencia en el crecimiento económico", Información Comercial Española, ICE, Revista de Economía, 823, pp. 131-140 (www. revistasice.com).

COLEMAN, J. S. (1994), "Social Capital", in J.S. Coleman, Foundations of Social Theory, Cambridge, Belknap. 
DIARIO DE FERROL (2006), "Los vecinos limpian la calle San Francisco de escombro", in Diario de Ferrol, 8 de abril de 2006.

DIARIO OFICIAL DE GALICIA, 1981-2006, varios números.

INE, Censo de Población y Viviendas, 2001 (www. ine.es).

INE, Padrón Municipal de Habitantes, 2006 (www. ine.es).

MILLÁN, R. e S. Gordon (2004), "Capital social: una lectura de tres perspectivas clásicas", Revista Mexicana de Sociología, 66, 4, p. 725.
OFICINA DE PLANEAMIENTO (2000), Memoria del área de rehabilitación para los barrios históricos de Ferrol Vello-Magdalena, Ferrol.

OFICINA DE REHABILITACIÓN DE FERROL: Fichas de las solicitudes de subvención, 2002-2006, Ferrol.

PUTNAM, R. D. (ed.) (2003), El declive del capital social. Un estudio internacional sobre las sociedades y el sentido comunitario, Barcelona, Círculo de lectores.

\section{Internet}

Plataforma Ártabra 21: http://artabra21.blogspot.com.

Asociación de Ferrol Vello: http://www.jazztelia.com/ ferrol-vello/blog. 